Original Research Article

\title{
A comparative study on the pollen viability and storage conditions of the nuclear and non - nuclear varieties
}

\author{
Shasha Tang, Yuling Huang,Xiao Fu \\ College of Plant Science and Technology, Wuxi Agricultural University, Jiangsu, China
}

\begin{abstract}
The fresh dried pollen of grape and seedless grape varieties were used as the research material. Each cultivar was stored at room temperature, $5 \mathrm{C}, 0 \mathrm{C},-18 \mathrm{C}$ and $-40 \mathrm{C}$ respectively. Sucrose $200 \mathrm{~g} / \mathrm{L}+$ boric acid $50 \mathrm{mg} / \mathrm{L}+\mathrm{agar}$ $8 \mathrm{~g} / \mathrm{L}$ as the nutrient substrate, and the comparative study on the germination and culture of the nuclear breed and the seedless cultivar. The results showed that the pollen viability decreased with the increase of storage time at different temperatures. The pollen viability decreased at $-18 \mathrm{C}$ and -40 , and the pollen viability decreased at the same temperature The There was a significant difference in pollen viability between different cultivars at the beginning of storage, and the pollen viability of the kernel breed was higher than that of the seedless grape.
\end{abstract}

KEYWORDS: Benzoxazine; Dielectric properties; Copolymerization; Modification

\section{Introduction}

Grape is a grape variety (Vitis) plant, seedless grape breeding is the world's grape breeding work of the important goal, as people's living standards continue to improve, seedless grapes in fresh and dried have shown show more more important role in the international market, the largest and most popular fresh and dried varieties are mainly seedless grape varieties. At present, the breeding of high quality non-nuclear grape varieties has become at home and abroad, especially in developed countries. Pollen has genetic information in the sexual production, early collection, storage of pollen and determination of pollen viability are the basis of artificial hybrid pollination. Pollen life is very short, but save them under a special condition may extend their life. [1]. There are differences in the life, storage conditions and viability of different plant pollen under natural conditions. Which pollen germination rate is one of the indicators to measure the size of pollen viability, also play as the most important indicators $[2,3]$.

In China's traditional seedless grape breeding, mostly with nuclear varieties for the female and non-nuclear varieties of hybrid, and this is currently the most widely used Chinese breeders and seedless grape breeding methods, and in the cross breeding of the non-nuclear In the important way of grape selection, pollen viability is an important condition for ensuring the success of hybrid breeding. As the flowering of different grape varieties is often inconsistent, in the breeding of seedless breeding process, often involved in pollen collection and pollen storage problems [4]. There are more studies on pollen viability [5-10], but the study of grape pollen viability and storage conditions is not too much [11-13], especially on the viability of nuclear and seedless grape pollen the study. Therefore, it is very important to study the viability and storage conditions of seedless and variety of grape pollen. The effects of different storage temperature and time on the pollen viability of different varieties and the pollen viability of different varieties under different conditions were studied in order to provide the theoretical basis for short - term storage and cross breeding of grape pollen.

\section{Materials and methods}

\subsection{Materials}

Test materials are from May 13, 2013 from the Chinese Academy of Agricultural Sciences Zhengzhou fruit trees, total of 10 varieties: nuclear varieties for the aromatic La Chagaki, Ze Yu, Jingxiu, Zhengzhou early red, Rui Beier; nonnuclear breeds are Himmler, the Amethyst, 8611, 8612. In the early flowering stage of grapes choose to grow robust

Copyright (C) 2017 -. This is an Open Access article distributed under the terms of the Creative Commons Attribution-NonCommercial 4.0 International License (http://creativecommons.org/licenses/by-nc/4.0/), permitting all non-commercial use, distribution, and reproduction in any medium, provided the original work is properly cited. 
plants, mining under the development of normal inflorescence, respectively, into the preservation bag, and then into the incubator with ice bag back to the laboratory.

\subsection{Pollen collection}

Will be brought back to the laboratory of the inflorescence, remove the top of the poor development of the buds, remove the anthers, placed in the pad with sulfuric acid paper covered dishes, remove the impurities and broken anthers, and then placed in the shade dish hand dry place and let them dry naturally, at room temperature. After a certain period of time, the pollen will be cracking, until the pollen is completely drained and completely dry and then pour into the mortar grinding. Through filtered by the small wire filter, they can collect them.

\subsection{Storage conditions for pollen}

The pollen of each variety was collected into 5 equal parts, respectively, into the centrifuge tube, the nozzle with a sealed seal, and then were placed in a dryer with calcium chloride sealed, and then sub-packaging. Good different varieties of pollen, were marked at room temperature, respectively, $5 \mathrm{C}, 0 \mathrm{C},-18 \mathrm{C},-40 \mathrm{C}$ under the conditions of shading storage.

\subsection{Test methods}

The germination rate of pollen was determined by in vitro germination. The pollen viability of the grape pollen was determined by using the solid medium of sucrose $200 \mathrm{~g} / \mathrm{L}+50 \mathrm{mg} / \mathrm{L}+$ agar $8 \mathrm{~g} / \mathrm{L}$ concentration as the nutrient substrate. The germination rate of fresh dried pollen was determined before storage, and the pollen germination rate was taken as the pollen viability index.

\subsubsection{Cultivation of pollen}

The medium was set to the medium according to the above formula. The $\mathrm{pH}$ was adjusted to 6.0 to 6.5 , and the medium was poured into the culture dish. After the medium was cooled and solidified, the cotton was dipped with a small amount of pollen and spread evenly on the surface of the medium. Under the microscope to observe whether the spread evenly, if the pollen distribution evenly, and then pad the wet filter paper tray covered with it, and finally labeled with the label, processing, planting time, placed in 25 under the conditions of incubator Each processing is set to 3 repetitions.

\subsubsection{Observe and record the results}

After 8 hours of culture, pollen germination was observed under $10 \times 20$ optical microscope. The germination rate was observed. Three treatments were randomly observed for each treatment. Each field of view was not less than 50 pollen, and the number of pollen germination per field and Pollen total grain number were recorded and take pictures, then calculate the pollen germination rate, take the mean. Pollen germination criteria: pollen tube length more than pollen grain diameter of $1 / 2$ for the germination pollen. Pollen germination rate formula:

Pollen germination rate $(\%)=$ number of germination pollen grains per field / total number of pollen in the field of view $\times 100 \%$.

Test results data processing using Excel software for mapping and analysis.

\section{Results and analysis}

\subsection{Comparison of Vinegar Viability with Nuclear Varieties and Non - nuclear Varieties}

As shown in Fig. 1, the average germination rate of pollen of the grape varieties before storage was $50.41 \%$ of the early red in Zhengzhou, $46.22 \%$ of the aromatic scabard, $43.04 \%$ of the rabbits, $40.35 \%$ of Zeyu, $14.13 \%$ of Jingxiu, The average germination rate of the pollen of the grape was $24.51 \%$ of Hildeau and $31.09 \%$ of the Amethyst, respectively. Among them, there were no pollen in the three seedless varieties of Tachikawa, 8611 and 8612 , but the pollen germination rate was 0. Pollen morphology for the dry, irregular shape shrinkage, 8611 and 8612 two nonnuclear varieties of pollen is round full, normal development, are pollen abortion. The germination order of the pollen germplasm before the storage was as follows: Zhengzhou early red $>$ aromatic pulla $>$ Rebel $>\mathrm{Ze} \mathrm{Yu}>$ Beijing Amethyst $>$ Hilmao De> Jingxiu> Tachikawa no nuclear, 8611,8612.

As shown in Fig. 2, the pollen germination rate of each cultivar at different storage temperatures after $5 \mathrm{~d}, 10 \mathrm{~d}$, $15 \mathrm{~d}, 20 \mathrm{~d}$ and $25 \mathrm{~d}$ storage, the pollen germination rate of the cultivars was gradually increased with the increase of 
storage time, But the pollen germination rate of different varieties of pollen decreased. The same storage time, storage temperature under the nuclear varieties of grape resistance to storage capacity than non-nuclear varieties is better. Therefore, from the pollen germination situation, the pollen germination rate of the grape varieties is higher than that of the non-seeded varieties, that is, the pollen viability is higher than that of the seedless varieties.

\subsection{Effects of Different Storage Temperatures on Pollen Viability of Grape}

\subsubsection{Effects of Different Storage Temperatures on Pollen Viability of Nuclear Grape Varieties}

It can be seen from Fig. 3 that the pollen germination rate of each cultivar is relatively high when the grape has been stored, but the germination rate of pollen germination rate decreases with the storage time prolonged. Under the condition of normal temperature, the pollen viability decreased with the increase of storage time, and the pollen germination rate of each cultivar decreased by nearly half after 10 days. The average germination rate of pollen in three varieties of Jingxiu, Zhengzhou early red and Rebecer was 7.64\%, 9.41\% and 7.38\% respectively after 25 days of storage. It can be seen that under normal temperature, pollen effect is poor. But if a short period of time for pollen storage, the effect is still ideal, and more convenient. Compared with the storage time, the pollen with 5C and 0C increased with the increase of storage time. The average germination rate of pollen after storage for 25 days was about $14.54 \%-25.12 \%$.

When the storage of the grape pollen was stored at $-18 \mathrm{C}$ and $-40 \mathrm{C}$, the germination rate of each pollen decreased slowly with the increase of storage time. The descending rate of the pollen was $5 \mathrm{C}, 0 \mathrm{C}$. The pollen germination rate under storage conditions was small, and the germination rate of pollen was maintained at more than $20 \%$ after 25 days of storage. For example, aromatic scab group could be maintained at $29.97 \%$ and Rebil could be maintained at $29.88 \%$. It can be seen, in this low temperature conditions, the storage effect is better, suitable for long storage of pollen.

\subsubsection{Effects of Different Storage Temperatures on Pollen Viability of Seedless Grape Varieties}

The seed germination rate of the five seedless varieties was 0.0 , and the pollen germination rate of the three seedless varieties was 0.0 , and the pollen morphology of Tachikawa was dry and the shape was shrunk irregularly. , 8611 and 8612 two non-nuclear varieties of pollen is round full, normal development but no pollen germination, are pollen abortion. The germination rate of pollen germination rate decreased with the storage time at different storage temperatures (Fig. 4). It can be seen from Fig.4 that the germination rate of fresh pollen of two non-nuclear cultivars before Himmler and Jing Ametha were $26.51 \%$ and $31.09 \%$ respectively, but the germination rate of pollen after storage for 15 days under normal temperature was lower $5 \%$, and the pollen viability of the Amethyst was almost 0 . At $5 \mathrm{C}$, the pollen germination rate decreased at $0 \mathrm{C}$, and the pollen germination rate decreased at $-18 \mathrm{C}$ and $-40 \mathrm{C}$ The speed of the slowest. It can be seen that the storage temperature of the grapevine with the nuclear variety is similar to that of the storage time. The effect of the storage of the seedless varieties with good temperature at $-18 \mathrm{C}$ and $-40 \mathrm{C}$ is better at $5 \mathrm{C}$ and $0 \mathrm{C}$. , At room temperature storage pollen effect is poor.

\section{Discussion}

The results showed that the pollen viability decreased with the increase of storage time, and the pollen viability decreased at $-18 \mathrm{C}$ and $-40 \mathrm{C}$ under the condition of the grape or the seedless grape. The most slowly, 5C, 0C storage conditions, followed by normal temperature storage conditions, the most rapid decline in pollen viability. In addition, there was a significant difference in the pollen viability between the different cultivars and the fresh pollen viability among the different cultivars before storage. The average germination rate of pollen was $50.41 \%$ in Zhengzhou, $46.22 \%$ in aroma and $46.04 \%$ in Rebel, $40.35 \%$ in Zeyu, 24.13\% in Jingxiu, and pollen of non-seeded grape. The average germination rates were $26.51 \%$ of Hildeau and $31.09 \%$ of Amethyst, respectively. Among them, there were no pollen but no pollen germination rate of 0 . In general, the pollen viability of the nuclear breed grape is higher than that of the seedless grape. In addition, there are pollen germination rate with time changes have a short time or a constant trend, such as the nuclear variety Ze jade and seedless varieties of Beijing amethyst, in -18C storage conditions, Ze Yu 20 days after storage Pollen viability higher than 15d, Beijing amethyst is in these two periods of pollen viability was flat state, it may be because each time the pollen germination rate, the sealed cap open to make it with the air Contact, change the bottle of humidity and oxygen concentration, and enhance the metabolism of pollen, so that its vitality increased within a short time; may also be due to alternating hot and cold, to stimulate the germination of pollen; there may be varieties of genes determined Of the specific reasons for further study.

There are a number of reasons for the rapid decline in pollen viability, some people think that pollen is very strong due to material metabolism; some people think that with the dry conditions; some people think that with the protein metabolism. The same point of pollen aging is the depletion of respiratory matrix, enzyme inactivation, dry injury and metabolic blockage [14]. Pollen is to maintain the vitality of the length, on the one hand by the genetic factors of the decision, on the other hand also affected by environmental factors [2]. The pollen viability of different varieties of grapes 
decreased with the increase of storage time. When pollen storage, with the increase of storage time, the consumption of storage material in pollen increased, the activity of enzyme decreased, the water gradually lost, the pollen viability will gradually decrease [15].

Pollen storage temperature and humidity are the main factors that affect the length of pollen storage. Pollen in high temperature, high humidity, the enzyme hydrolysis activity increased, the metabolic effect also will be strengthened, and carried out a strong respiration, consumption of large amounts of nutrients, and susceptible to microbial infection moldy loss of germination rate [7]. In the process of pollen storage, the low temperature of the environment can be reduced, the metabolism can be inhibited, the activity of the enzyme is reduced, the respiration is reduced, the pollen vitality is maintained for a long time, and the life of the storage pollen is often negatively correlated with the relative humidity [16] The results showed that the pollen viability was the best at the temperature of $-18 \mathrm{C}$ and $-40 \mathrm{C}$, and the pollen viability decreased gradually with the prolonging of storage time. The main reason was that the pollen nutrient gradually consumed and the enzyme activity decreased gradually. The pollen viability decreased most rapidly at room temperature, mainly due to the high storage temperature, strong activity in pollen, accelerated metabolism, excessive consumption of nutrients stored in pollen, and $5 \mathrm{C}, 0 \mathrm{C}$ conditions between the two, is the most convenient storage of short-term storage.

Of the 10 grape varieties tested, it was found that the pollen viability of the grape varieties was higher than that of the seedless varieties, but the germination rate of the fresh pollen was not too high, and the ridge of the population was 8611,8612 The pollen germination rate was zero, and the pollen germination rate was undisturbed and the shape was shrunk irregularly. The pollen of the two seedless varieties of 8611 and 8612 was round and full, and the pollen germination rate was zero., belonging to pollen abortion, the specific reason remains to be studied.

\section{References}

1. Ren Qiuping, Zhang Binbin. Effects of Different Storage Temperatures on Pollen Viability of Several Pear Cultivars [J]. Northern Horticulture, 2008 (3): 3-5.

2. YIN Jia-lei, ZHAO Hui-yen. Effects of pollen viability and pollen storage [J]. CHINESE AGRICULTURAL SCIENCE BULLETIN, 2005,21 (4): 110-113,193.

3. Wang Qinli, Lu Long Dou, Wu Xiaoqin, et al. Preservation of pollen and its viability [J]. Chinese Journal of Botany, 2002,19 (3): 365-373.

4. Xu Yuejin, Hu Chungen, editor. Horticultural plant breeding [M]. Higher Education Publishing House .2007, : 23-85.

5. Acta Physica Sinica, 2007,43 (1): 181-183.

6. ZHAO Cai-ping, LIU Na, HAN Ming-yu, et al. Effects of different storage temperatures on peach pollen viability [J]. Northern Horticulture, 2010 (12): 50-52.

7. Liu Huichao, Jia Wenqing, Liu Luping, et al. Effects of different conditions on pollen viability in Yingchun [J] .Guangdong Agricultural Sciences, 2011 (6): 55-57.

8. Song Hongxia, Hou Lufen, Zhang Guangxing, et al. Effects of storage temperature and humidity on pollen viability of carrots [J]. Journal of Shanghai Agricultural University, 2011,27 (1): 65-67.

9. Liu Zigang. Effects of different storage temperatures on pollen viability and pollination and fruiting ability of platycodon grandiflorum $[\mathrm{J}]$

10. Ma Jie. Effects of different storage temperature and time on peach pollen viability [J] .Jilin Agricultural Sciences .2013,38 (5): 73-76

11. Yang Rui, Hao Yan, Wang Faolin, Zhang Cunzhi, et al. Determination of Vinegar Viability [J]. Journal of Gansu Agricultural University, 2007,42 (2): 47-50.

12. Xue Xinwei, Ma Zuotu, Wu Longlong, et al. Grape pollen storage test [J]. Agricultural Information and Technology .2012. (22): 18-19,31.

13. Li Guirong, Zhu Ziqiu, Li Li, Cai Zuguo, et al. Experimental study on the germination of pollen seedless pollen in vitro [J]. Journal of Northwest Forestry University, 2012, 27 (6): 93-97.

14. Zhu Jun, Genetics [M]. Beijing: China Agricultural Publishing House .2002: 112-113.

15. Pan Ruichang, Plant Physiology [M]. Beijing: Higher Education Press .2001: 257-259.

16. Song Hongxia, Hou Lufen, Zhang Guangxing, et al. Effects of storage temperature and humidity on pollen viability of carrots [J]. Journal of Shanghai Agricultural University, 2011,27 (1): 65-67. 